\title{
Does double high-level disinfection for duodenoscopes add any value? A mini- systematic review and meta-analysis
}

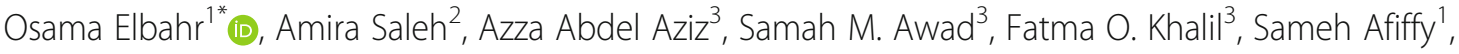 \\ Ahmad Kamal', Ayman Alsebaey ${ }^{1}$ and Gamal Badra ${ }^{1}$
}

\begin{abstract}
Background: Duodenoscope-emerging infection especially drug-resistant bacteria is considered a major concern nowadays. Different approaches were attempted to overcome this problem, like double high-level disinfection procedure. We performed a systematic review and meta-analysis to evaluate risk difference for positive cultures from duodenoscopes between double high-level disinfection (dHLD) and single (standard) high-level disinfection (sHLD).
\end{abstract}

Main body: A thorough literature search (in October and November 2019) for studies comparing dHLD and SHLD for duodenoscopes was performed by 3 researchers in the Web of Science, Scopus, PubMed, and Cochran databases. The search terms were "duodenoscope," "ERCP endoscope," "disinfection," "sterilization," and "reprocessing," and only randomized clinical trials with the English language were accepted.

Four trials were identified studying $\mathrm{dHLD}$, and only 2 clinical trials comparing $\mathrm{dHLD}$ with standard SHLD were found reporting 6193 duodenoscope cultures. Overall sHLD cultures were 2972, and dHLD cultures were 3221; overall positive cultures were 140 in SHLD and 161 in dHLD.

The results of a meta-analysis using the random-effect model showed no significant risk difference (RD) between the 2 procedures for duodenoscope positive cultures ( $p=0.53, \mathrm{RD} 0.003,95 \% \mathrm{Cl}$ " $-0.007-0.013^{\prime \prime}$ ).

Conclusions: Double HLD offered no significant difference over single HLD for duodenoscope disinfection. An alternative strategy to overcome duodenoscope-transmitted infection is a big issue to be resolved.

Keywords: Duodenoscope, ERCP, Disinfection, High-level, MDRO, CRE, Culture, Review, Meta-analysis, Bacteria

\section{Background}

Gastrointestinal (GI) endoscopy procedures are performed each year worldwide. Gastroenterologists and surgeons use flexible endoscopes for providing minimally invasive diagnostic, therapeutic, and palliative care, and the overall low rates of associated adverse events have offered such procedures optimal risk-benefit ratio [1]. The duodenoscope, a complex endoscopic instrument, is frequently utilized to accomplish the increasing demand for minimally invasive treatments of pancreatic

\footnotetext{
*Correspondence: oelbahr@yahoo.com; osama.elbahr@liver.menofia.edu.eg ${ }^{1}$ Hepatology and Gastroenterology, National Liver Institute, Menoufia University, Menoufia, Egypt

Full list of author information is available at the end of the article
}

and biliary diseases. It is, besides, the fundamental tool used to perform endoscopic retrograde cholangiopancreatography (ERCP) [2] for biliary drainage [3, 4].

Side-viewing endoscopes (duodenoscopes used for ERCP) have an additional unique feature, a cantilevered elevator mechanism at the distal tip controlled by an elevator wire (via a dedicated channel). This design is challenging to clean and disinfect and has been proposed as a contributing factor to recent outbreaks associated with duodenoscopes [5]. Severe infectious complications occur after ERCP in 2-4\% of patients and include bacteremia, ascending cholangitis, cholecystitis, liver abscess, and necrotizing pancreatitis. Infection risk is higher when the biliary obstruction is present versus

\section{Springer Open}

(c) The Author(s). 2020 Open Access This article is licensed under a Creative Commons Attribution 4.0 International License, which permits use, sharing, adaptation, distribution and reproduction in any medium or format, as long as you give appropriate credit to the original author(s) and the source, provide a link to the Creative Commons licence, and indicate if changes were made. The images or other third party material in this article are included in the article's Creative Commons licence, unless indicated otherwise in a credit line to the material. If material is not included in the article's Creative Commons licence and your intended use is not permitted by statutory regulation or exceeds the permitted use, you will need to obtain permission directly from the copyright holder. To view a copy of this licence, visit http://creativecommons.org/licenses/by/4.0/. 
absent and when an obstruction is malignant versus benign. Sepsis after ERCP has a mortality rate as high as $29 \%[6,7]$.

Duodenoscopes undergo multistep cleaning and a high-level disinfection procedure called reprocessing so that they can be reused among patients. The complex design of duodenoscopes impedes effective cleaning [8]. Duodenoscopes require high-level disinfection (HLD) using chemical disinfectants. Glutaraldehyde, hydrogen peroxide, ortho-phthalaldehyde (OPA), peracetic acid, peracetic acid with hydrogen peroxide, and a chlorinebased system are cleared by the FDA and are dependable high-level disinfectants which provided the factors influencing that germicidal procedures are met [9].

This mini-systematic review evaluates the efficacy of double high-level disinfection (dHLD); dHLD was a possible sterilization method to overcome this problem by performing 2 cycles of the automatic standard disinfection process over standard high-level disinfection (sHLD) on the decrease of positive duodenoscope culture rates.

\section{Main text}

\section{Search methodology}

A thorough literature search (in October and November 2019) for studies comparing dHLD and sHLD for duodenoscopes was performed in the Web of Science, Scopus, PubMed, and Cochran databases. The search terms were "duodenoscope," "ERCP endoscope," "disinfection," "sterilization," and "reprocessing."

\section{Criteria of selected studies Types of studies}

Clinical trials in the English language that study dHLD were included.

\section{The type of participant}

Humans with a hepatobiliary indication for ERCP intervention were included.

\section{The type of intervention}

dHLD means 2 cycles of automated duodenoscope sterilization while sHLD means 1 cycle of standard highlevel disinfection.

\section{Study selection}

Studies were independently evaluated by five authors (E.O., A.S., S.A., AL.A., and K.A.) who screened the title, the abstract, and then the full text, with articles being removed according to the exclusion criteria at each step, and this was done by the aid of Rayyan web. To ensure that all potentially relevant studies were considered for inclusion, further evaluation by a consensus of 4 authors (B.G., AB.A., AW.S., and K.F.) was performed.

\section{Data extraction and risk of bias assessment}

By 2 authors, the following data were extracted from each study: the first author, the year of publication, the study design, the number of participants, the type of intervention, the number of positive cultures, and the presence of drug-resistant organisms.

The risk of bias was conducted on RevMan 5 software.

\section{Primary outcome}

The primary outcome is culture-positive duodenoscopes after disinfection.

The percentage of the events were used to measure the treatment effect.

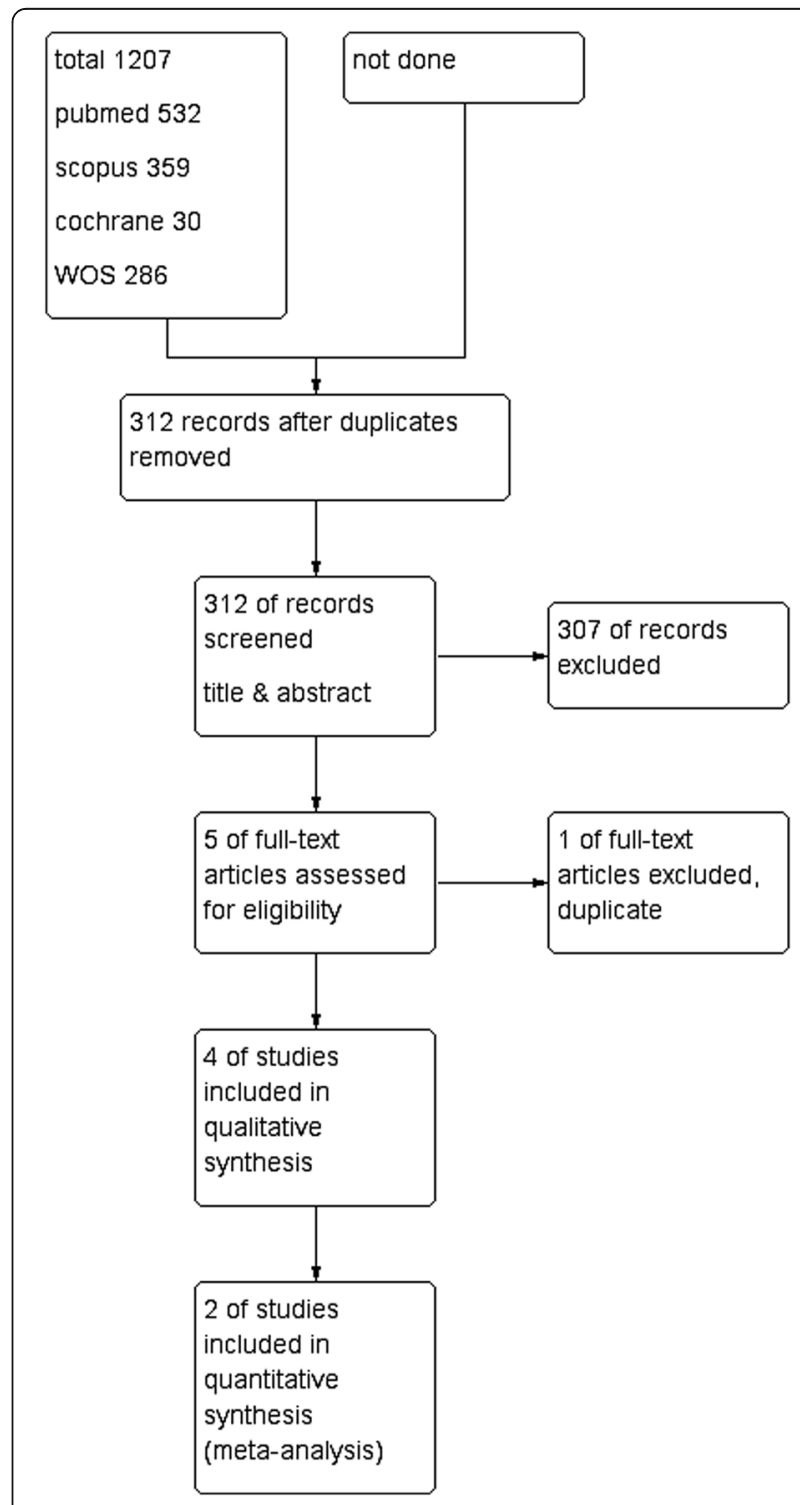

Fig. 1 Prisma flowchart for studies selection 


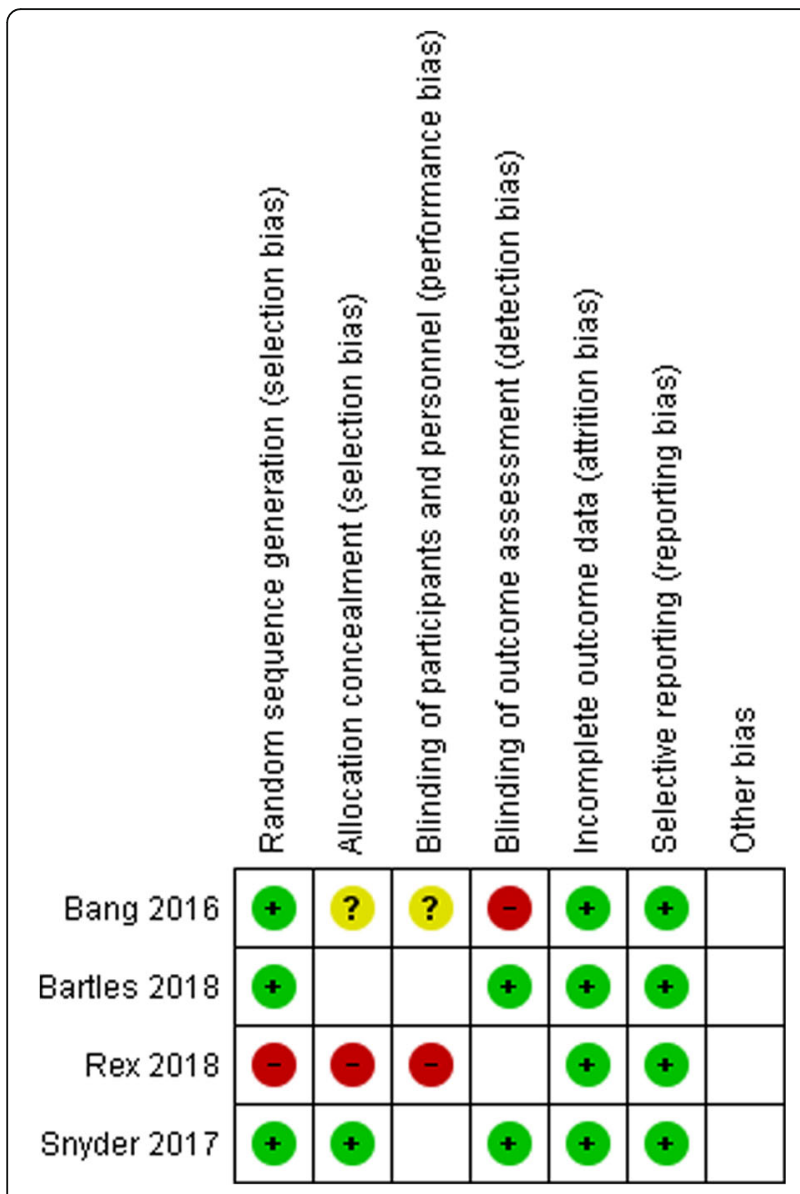

Fig. 2 Risk of bias for selected studies

\section{Statistical analysis}

All analysis was conducted on RevMan 5 software.

A meta-analysis with $95 \%$ confidence interval (CI) was performed to measure risk difference in culture-positive duodenoscopes between dHLD and sHLD for included and eligible studies, and a random-effect model was applied.

\section{Results}

$X^{2}$ test was used to assess the presence of heterogeneity, while $I^{2}$ test was used to assess the degree of heterogeneity.
A total of 1207 records were retrieved from databases based on search criteria (Fig. 1). After reviewing the title, abstracts, and full texts, 4 studies were found to be eligible for synthetic narrative analysis while only 2 were eligible for meta-analysis (Snyder et al. [10] and Bartles et al. [11]) as they compared dHLD against sHLD. A total of 6193 duodenoscope disinfection processes were found in the 4 studies. sHLD was presented in 2 studies (Snyder et al. [10] and Bartles et al. [11]) conducted 2972 times, while dHLD was presented in 4 studies done 3221 times.

Bang et al. [12] and Rex et al. [13] were single-arm studies evaluating dHLD. Snyder et al. [10] and Bartles et al. [11] (with the highest weight in our meta-analysis 98.6\%) conducted a comparative clinical trial between dHLD and SHLD. So, this is presented in the risk of bias assessment in Fig 2.

There was no significant heterogeneity between the studies $\left(\chi^{2}=0.7\right.$ and $I^{2}=0 \%$ ) (Fig. 3).

The primary outcome is shown in Table 1 and Fig. 3.

Positive culture rate in sHLD was $4.7 \%$ while in dHLD was $4.9 \%$, and there is no significant risk difference (RD) between both $(p=0.53$, RD 0.003 , 95\% CI “- $0.007-$ $0.013 ”)$.

Duodenoscope-related infections are of increasing global concern due to the emergence of multidrugresistant bacteria such as carbapenem-resistant Enterobacteriaceae (CRE), with a bacterial biofilm production postulated as one cause of persistent infection from such virulent organisms [14]. The current standard for all flexible endoscope reprocessing includes pre-cleaning, leak testing, an additional manual cleaning step, and high-level disinfection [15]. Different disinfection alternative methods, over the standard one, were tried, such as $N$-acetylcysteine (NAC) [14], plasma-activated water (PAW) [16], and dHLD.

Our systematic review and meta-analysis tried to evaluate the efficacy of dHLD over SHLD, but we have been faced by a small number of trials on such topic. Snyder in his trial terminated the study after the followup period due to failure to reach the primary outcome of his study (multidrug-resistant organisms [MDRO]),

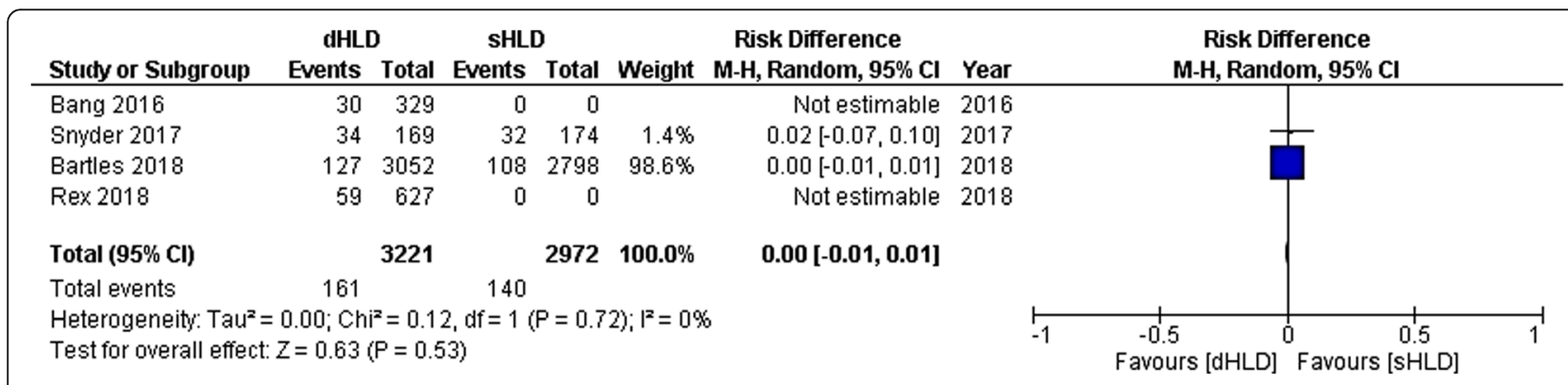

Fig. 3 Forest plot of comparison: risk difference for a positive culture 
Table 1 General data

\begin{tabular}{ll}
\hline Total number of studies included & 4 \\
\hline Total number of duodenoscopes processed & 6193 \\
SHLD positive cultures/total & $140 / 2972$ \\
dHLD positive cultures/total & $161 / 3221$ \\
\hline
\end{tabular}

but he concluded that AHLD did not decrease the culture-positive rate in duodenoscopes over sHLD. Bartles has conducted his study on both duodenoscopes and endoscopes and found that double HLD did not reduce culture positivity rates compared with single HLD in facilities with an already low positive culture rate.

All that was presented in our meta-analysis showed no statistically significant RD between dHLD and sHLD ( $p=0.53$, RD $0.003,95 \%$ CI “- 0.007-0.013”).

\section{Conclusion}

What are the implications of our review for the future? Our analysis showed that dHLD had no added value over the standard HLD. Therefore, another strategy should be adopted to overcome the duodenoscopetransmitted infections and quality measures should be ascertained for the following guidelines of duodenoscope disinfection.

\section{Abbreviations}

dHLD: Double high-level disinfection; sHLD: Single high-level disinfection; ERCP: Endoscopic retrograde cholangiopancreatography; MDRO: Multidrugresistant organisms; CRE: Carbapenem-resistant Enterobacteriaceae; $X^{2}$ : Chisquare test

\section{Acknowledgements}

To all our professors

\section{Authors' contributions}

O.E: literature search, study selection, data extraction, bias assessment, review writing, and statistical analysis. A.S: literature search, study selection, review writing, data extraction, and bias assessment. S.A: literature search and study selection. A.A: literature search, study selection, and critical appraisal. A.K: literature search and study selection. G.B: resolve disagreement and critical appraisal. A.A.A: resolve disagreement and critical appraisal. S.M.A: resolve disagreement and critical appraisal. F.K: resolve disagreement and critical appraisal. All authors read and approved the final manuscript.

\section{Funding}

No funding

\section{Availability of data and materials}

Not applicable

\section{Ethics approval and consent to participate}

Not applicable

\section{Consent for publication}

Not applicable

\section{Competing interests}

No competing interests

\section{Author details}

${ }^{1}$ Hepatology and Gastroenterology, National Liver Institute, Menoufia

University, Menoufia, Egypt. ${ }^{2}$ Infection Control, Student Hospital, Menoufia
University, Menoufia, Egypt. ${ }^{3}$ Medical Microbiology, and Immunology, National Liver Institute, Menoufia University, Menoufia, Egypt.

Received: 16 March 2020 Accepted: 8 June 2020

Published online: 17 August 2020

References

1. Balan GG, Sfarti CV, Chiriac SA, Stanciu C, Trifan A (2019) Duodenoscopeassociated infections: a review. Eur J Clin Microbiol Infect Dis. https://doi. org/10.1007/s10096-019-03671-3

2. Higa JT (2019) Duodenoscope-related infections: overview and epidemiology. Tech Gastrointest Endosc. https://doi.org/10.1016/j.tgie.2019. 150623

3. Elbahr O, Zakaria H, Zakaria HM et al (2018) Impact of preoperative endoscopic biliary drainage on postoperative outcome after pancreaticoduodenectomy. Surgery, Gastroenterol Oncol 23:3. https://doi. org/10.21614/sgo-23-2-173

4. Elbahr O (2019) Biliptysis. Egypt liver J 9. https://doi.org/10.1186/s43066-0190005-8

5. Grein JD, Murthy RK (2018) New developments in the prevention of gastrointestinal scope-related infections. Infect Dis Clin N Am 32(4):899-913. https://doi.org/10.1016/j.idc.2018.06.008

6. Kovaleva J, Peters FTM, van der Mei Mei HC, Degener JE (2013) Transmission of infection by flexible gastrointestinal endoscopy and bronchoscopy. Clin Microbiol Rev 26(2):230-253. https://doi.org/10.1128/CMR.00085-12

7. Chandrasekhara V, Khashab MA, Muthusamy VR et al (2017) Adverse events associated with ERCP. Gastrointest Endosc 85(1):32-47. https://doi.org/10. 1016/j.gie.2016.06.051

8. Chang W-K, Liu T-C, Liu T-L, Peng C-L, Wang H-P (2019) Enhanced manual cleaning efficacy of duodenoscope in endoscopy units: results of a multicenter comprehensive quality control program. Am J Infect Control 47(10):1233-1239. https://doi.org/10.1016/j.ajic.2019.03.029

9. Rutala WA, Weber DJ (2019) Reprocessing semicritical items: outbreaks and current issues. Am J Infect Control 47:A79-A89. https://doi.org/10.1016/j.ajic. 2019.01.015

10. Snyder GM, Wright SB, Smithey A et al (2017) Randomized comparison of 3 highlevel disinfection and sterilization procedures for duodenoscopes. Gastroenterology. 153(4):1018-1025. https://doi.org/10.1053/j.gastro.2017.06.052

11. Bartles $R L$, Leggett JE, Hove $S$ et al (2018) A randomized trial of single versus double high-level disinfection of duodenoscopes and linear echoendoscopes using standard automated reprocessing. Gastrointest Endosc 88(2):306-313.e2. https://doi.org/10.1016/j.gie.2018.02.016

12. Bang JY, Rex DK, Sherman S et al (2016) Impact of implementation of double-reprocessing protocol in the prevention of duodenoscopeassociated carbapenem-resistant Enterobacteriaceae (CRE) in a single tertiary referral center. Gastrointest Endosc 83(5):AB170. https://doi.org/10. 1016/j.gie.2016.03.144

13. Rex DK, Sieber M, Lehman GA et al (2018) A double-reprocessing high-level disinfection protocol does not eliminate positive cultures from the elevators of duodenoscopes. Endoscopy. 50(6):588-596. https://doi.org/10.1055/s0043-122378

14. Kwok K, Chang J, Lo S, Giap A, Lim B, Wu B (2016) A novel adjunctive cleansing method to reduce colony-forming units on duodenoscopes. Endosc Int open 4(11):E1178-E1182. https://doi.org/10.1055/s-0042-116490

15. Kim S, Muthusamy VR (2016) Current practice of duodenoscope reprocessing. Curr Gastroenterol Rep 18(10):54. https://doi.org/10.1007/ s11894-016-0528-7

16. Balan GG, Rosca I, Ursu E-L et al (2018) Plasma-activated water: a new and effective alternative for duodenoscope reprocessing. Infect Drug Resist 11: 727-733. https://doi.org/10.2147/IDR.S159243

\section{Publisher's Note}

Springer Nature remains neutral with regard to jurisdictional claims in published maps and institutional affiliations. 